\title{
Efeitos da fisioterapia durante o parto em um hospital da região amazônica: Um
}

\section{estudo piloto}

\author{
Effects of physiotherapy during labor in a hospital in the amazon region: A pilot study \\ Efectos de la fisioterapia durante el parto en un hospital de la región amazónica: Un estudio piloto
}

Recebido: 15/05/2021 | Revisado: 19/05/2021 | Aceito: 25/05/2021 | Publicado: 09/06/2021

Juliana de Jesus Balieiro

ORCID: https://orcid.org/0000-0002-0530-683X Universidade Federal do Pará, Brasil E-mail:julianabalieiro.13@gmail.com

Amanda Suzane Alves da Silva ORCID: https://orcid.org/0000-0002-5764-382X Universidade Federal do Pará, Brasil E-mail:suzane.amanda@gmail.com

Thalia Saraiva Mendonça

ORCID: https://orcid.org/0000-0002-7096-6367 Universidade Federal do Pará, Brasil E-mail:thaliasmendonca@gmail.com

Lúcio Flávio Garcia Rodrigues ORCID: https://orcid.org/0000-0002-8984-5856 Universidade Federal do Pará, Brasil E-mail:lucioflavio99@gmail.com

João Simão de Melo-Neto ORCID: https://orcid.org/0000-0002-4681-8532 Universidade Federal do Pará, Brasil E-mail:jsmeloneto@gmail.com

Marina Baia do Vale

ORCID: https://orcid.org/0000-0003-0003-721X Faculdade Paraense de Ensino, Brasil

E-mail: marinavalefisioterapeuta@gmail.com

Cibele Nazaré Câmara Rodrigues ORCID: https://orcid.org/0000-0002-2429-9970 Universidade Federal do Pará, Brasil

E-mail: cibelecamaranazare@gmail.com

\begin{abstract}
Resumo
Objetivo: Avaliar os efeitos da fisioterapia nas experiências positivas com o parto em um hospital na região Amazônica oriental. Método: Estudo piloto, intervencional, não-randomizado, com abordagem quantitativa e análises descritivas e inferenciais. Foram coletadas variáveis sociodemográficas, econômicas, histórico obstétrico e variáveis do parto; A experiência positiva no parto foi avaliada pela subescala do questionário de experiência e satisfação com o parto. Resultados: Participaram 43 parturientes, alocadas em grupo intervenção (GI) e grupo controle (GC); ambos os grupos apresentaram experiência positiva moderada; as mulheres que realizaram fisioterapia consideraram-se um membro útil e cooperativo com a equipe médica que a acompanhou; as primíparas no GI foram 80,9\% e no GC 54,5\%; observa-se que mulheres primíparas apresentaram associação com a presença de laceração (RR: 2,05 [IC 95\%: 1,08; 3,91]; $\mathrm{p}=0,007)$ e houve associação entre infecção do trato urinário inferior (ITU) e laceração (RR: 2,02; IC: 1,20; 3,$39 ; \mathrm{p}:=0,003$ ); mulheres que sofreram laceração tiveram um tempo de trabalho de parto maior (laceração(+): 18,50 horas [IC 95\%: 11,33; 39,79]; laceração(-): 6,00 [IC 95\%: -5,052; 33,95]; U: 78,00; p = 0,013). Conclusão: As mulheres que realizaram fisioterapia apresentaram como experiência positiva o fato de se considerarem um membro útil e cooperativo com a equipe médica que a acompanhou durante o parto. Além disso, observa-se que a primiparidade foi associada a laceração, sendo esta aumentada junto com o tempo de trabalho de parto e presença de ITU.
\end{abstract}

Palavras-chave: Fisioterapia; Trabalho de parto; Parto normal; Satisfação pessoal; Acontecimentos que mudam a vida.

\section{Abstract}

Objective: To evaluate the effects of physiotherapy on positive experiences with childbirth in a hospital in the eastern Amazon region. Method: Pilot, interventional, non-randomized study, with quantitative approach and descriptive and inferential analyses. Sociodemographic, economic, obstetric history and delivery variables were collected; The 
positive experience in childbirth was evaluated by the subscale of the questionnaire of experience and satisfaction with labor. Results: Participants were 43 parturients, allocated in an intervention group (GI) and control group (GC); both groups had moderate positive experience; the women who underwent physical therapy considered themselves a useful and cooperative member with the medical team that accompanied her; the primiparous women in GI were $80.9 \%$ and in GC 54.5\%; it is observed that primiparous women were associated with the presence of laceration (RR: 2.05 [95\% CI: 1.08; 3.91]; $\mathrm{p}=0.007)$ and there was an association between lower urinary tract infection (UTI) and laceration (RR: 2.02; CI: 1.20; 3,39; p: = 0.003); women who suffered laceration had a longer labor time (laceration(+): 18.50 hours [95\% CI: 11.33; 39.79]; laceration(-): 6.00 [95\% CI: -5.052; 33,95]; U: 78.00; $p=0.013$ ). Conclusion: The women who underwent physical therapy presented as a positive experience the fact that they considered themselves a useful and cooperative member with the medical team that accompanied her during childbirth. In addition, it is observed that primiparity was associated with laceration, which is increased along with the time of labor and presence of UTI.

Keywords: Physical therapy specialty; Labor, obstetric; Natural childbirth; Personal satisfaction; Life change events.

\section{Resumen}

Objetivo: Evaluar los efectos de la fisioterapia en experiencias positivas con el parto en un hospital de la Amazonía oriental. Método: Estudio piloto, intervencionista, no aleatorizado, con abordaje cuantitativo y análisis descriptivo e inferencial. Se recogieron variables sociodemográficas, económicas, antecedentes obstétricos y parto; La experiencia positiva en el parto se evaluó mediante la subescala del cuestionario de experiencia y satisfacción con el parto. Resultados: participaron 43 parturientas, asignadas al grupo de intervención (GI) y grupo de control (GC); ambos grupos tuvieron una experiencia positiva moderada; las mujeres que se sometieron a fisioterapia se consideraban un miembro útil y colaborador del equipo médico que las acompañaba; las primíparas en el GI fueron 80,9\% y en el GC 54,5\%; Se observa que las primíparas se asociaron con la presencia de laceración (RR: 2,05 [IC 95\%: 1,08; 3,91]; p = 0,007) y hubo asociación entre infección del tracto urinario inferior (ITU) y laceración (RR: 2,02 ; IC: 1,20; 3,39; p: = 0,003); las mujeres que sufrieron laceración tuvieron un mayor tiempo de trabajo de parto (laceración (+): 18,50 horas [IC 95\%: 11,33; 39,79]; laceración (-): 6,00 [IC 95\%: - 5,052; 33,95]; U: 78,00; p = 0,013). Conclusión: Las mujeres que se sometieron a fisioterapia presentaron como una experiencia positiva el hecho de considerarse un miembro útil y colaborador con el equipo médico que la acompañó durante el parto. Además, se observa que la primiparidad se asoció con laceración, la cual se incrementó junto con el tiempo de parto y la presencia de ITU.

Palabras clave: Fisioterapia; Trabajo de parto; Parto normal; Satisfacción personal; Acontecimientos que cambian la vida.

\section{Introdução}

Durante a gestação é gerada grande expectativa por parte da gestante e da família em relação aos eventos de trabalho de parto (TP) e parto. As mulheres apresentam expectativas de que sua atuação seja da forma mais independente possível, esperando manter os eventos sob seu controle e com segurança, embora reconheçam que este é um momento imprevisível. No início do TP e com o aumento das contrações uterinas, a mulher busca companhia, suporte emocional e segurança, sendo necessário a adoção de uma postura ética e solidária por parte dos profissionais. As maternidades devem receber a mulher com dignidade, juntamente com os seus familiares e o recém-nascido (RN), para isso a instituição deve organizar-se de maneira a criar um ambiente acolhedor e adotar condutas hospitalares que rompam com o tradicional isolamento imposto à mulher. (Downe et al., 2018; Ministério da Saúde, 2017)

Essa experiência pode influenciar de forma positiva ou negativa a curto ou longo prazo, principalmente nos aspectos psicológicos. Lembranças agradáveis dessa ocasião contribuem para a construção de memórias afetivas, repercutindo no bem estar da mulher. Da mesma forma, experiências negativas contribuem para comportamentos como medo de futuras gestações ou até mesmo pode resultar em doenças como a depressão pós parto (Nilvér et al., 2017). Repercussões negativas têm sido associada a fatores sociodemográficos, econômicos e obstétricos com prevalência entre mães jovens e acima de 35 anos, menor escolaridade, desempregadas e donas de casa, com gravidez não planejada, primiparidade, má avaliação da saúde materna e ocorrência de incontinência urinária (Abbasi et al., 2013). Contudo, as repercussões positivas e negativas necessitam ser mais discutidas, principalmente quando submetidas a algum tipo de tratamento que pode contribuir para o parto.

A fisioterapia visa auxiliar na atuação e proporcionar bem estar à parturiente. Dentre as suas atribuições, é responsável 
por aplicar intervenções não farmacológicas para o controle ou alívio da dor. Além disso, cabe ao fisioterapeuta, durante o TP, ajudar a mulher no posicionamento adequado, nos movimentos de mobilidade pélvica e no uso correto da musculatura diafragmática, com o objetivo de otimizar o TP. Tais condutas permitem melhor qualidade de parto, pois aumentam as chances de um parto sem grandes complicações, conforme preconizado no Programa de Humanização no Pré-natal e Nascimento (Baracho, 2018; Ministério da Saúde, 2017; Nakano et al., 2016). Neste sentido, estudos abordando o efeito da fisioterapia podem contribuir para uma prática baseada em evidência sustentada.

Portanto, o objetivo deste estudo é avaliar os efeitos da fisioterapia aplicada durante o TP sobre as experiências positivas com o parto em um hospital de referência em saúde materno infantil na região Amazônica oriental. Além disso, serão verificadas as variáveis: social, econômica, demográfica, uso de substâncias lícitas, comorbidade gestacional, desfechos do parto e dados antropométricos dos neonatos nos diferentes grupos.

\section{Metodologia}

\section{Aspectos éticos}

O estudo seguiu todos os preceitos éticos para pesquisa com seres humanos previstos na Resolução no 466/2012 e 510/2016 do Conselho Nacional de Saúde e foi aprovado pelo Comitê de Ética em Pesquisa da Universidade Federal do Pará (UFPA), parecer $\mathrm{n}^{\circ}$ 4.001.383. É importante salientar que as parturientes que aceitaram participar da pesquisa assinaram o Termo de Consentimento Livre e Esclarecido (TCLE).

\section{Tipo de estudo}

Estudo piloto, intervencional, não-randomizado, com abordagem quantitativa e análises descritivas e inferenciais (Estrela, 2018).

\section{Amostragem}

Amostragem foi do tipo probabilística aleatória simples.

\section{Participantes}

A amostra consistiu em 43 parturientes da maternidade do Hospital Santo Antônio Maria Zaccaria, localizado na cidade de Bragança-PA. A amostra foi estudada no período de setembro a novembro de 2020. As mulheres foram distribuídas entre grupo intervenção (GI) (n=21), parturientes admitidas no período diurno, e grupo controle (GC) (n=22) parturientes admitidas no período noturno. Para verificar o poder do teste frequentemente aplicado neste estudo (teste exato de Fisher) foram selecionados os dados referentes à laceração nos diferentes grupos experimentais. Proporção p1: 0,952; proporção p2: 0,363; erro $\alpha$ : 0,05 ; erro $\beta$ : 0,20; relação de alocação: 1 foram os parâmetros utilizados para determinar o poder atual: 0,85 . $O$ software GPower (Faul, 2007) foi usado para o cálculo.

\section{Critérios de inclusão}

Os critérios de inclusão foram: parturientes com gestação de risco habitual (caracterizada por ausência de síndromes hipertensivas, diabetes mellitus descompensada, obesidade, ruptura prematura das membranas, início prematuro de TP, gestação gemelar, descolamento prematuro de placenta, placenta prévia e incompetência istmo cervical), com idade gestacional superior a 37 semanas, de feto vivo, único e em apresentação cefálica, com início espontâneo de TP, que estavam na fase ativa do TP (duas contrações a cada 10 minutos e dilatação de 3 a 4 cm). 


\section{Critérios de exclusão}

Os critérios de exclusão foram: parturientes que realizaram acompanhamento fisioterapêutico no pré-natal, que possuíam alterações cognitivas e/ou psiquiátricas que não conseguissem responder às perguntas feitas pelo pesquisador e as que foram admitidas para indução do parto.

\section{Protocolo fisioterapêutico}

O protocolo fisioterapêutico consistiu em: deambulação assistida; exercício respiratório; massagem lombossacral; exercício de mobilidade pélvica na bola suíça; movimentos de inclinação lateral da pelve; e posicionamento.

A deambulação assistida foi incentivada a mulher a qualquer momento do TP, respeitando o nível de dor e a fadiga materna. Durante as contrações uterinas foram realizados os exercícios respiratórios, que consistiram de ciclos de respirações profundas, e a massagem lombossacral entre T10 e S4, a técnica empregada na massagem foi movimentos rítmicos circulares de amassamento com as mãos e deslizamento para as laterais da região do tronco associada a pressão sacral, a intensidade foi determinada pela parturiente, bem como a posição, podendo está sentada, decúbito lateral ou de pé, ambas as intervenções respeitaram o tempo de duração da contração uterina efetiva. O exercício de mobilidade pélvica na bola suíça foi utilizado com as parturientes que apresentavam 3 a $5 \mathrm{~cm}$ de dilatação do colo uterino e o feto estava acima do estreito médio ( -3 a -1 , De Lee), o fisioterapeuta incentivou a realização do exercício com a parturiente sentada na bola suíça movimentando a pelve a partir da posição neutra para a retroversão pélvica. Os movimentos de inclinação lateral da pelve foram realizados a partir de 6 a $7 \mathrm{~cm}$ de dilatação uterina e/ou quando o feto estava no estreito médio (quando alcança o plano zero de De Lee, no nível das espinhas isquiáticas), para facilitar a passagem do feto por esse local. Quando a parturiente alcançou 8 a $10 \mathrm{~cm}$ de dilatação uterina e/ou o feto estava abaixo do estreito médio $(+1 \mathrm{a}+3$, De Lee) foi realizado posicionamento para favorecer a anteversão pélvica. Durante o período expulsivo do TP foi incentivado o puxo espontâneo, porém, respeitando a escolha da parturiente para realização do puxo e posição de parto.

O GC não recebeu atendimento fisioterapêutico no TP e parto, recebeu somente prestação de serviços e cuidados usuais da equipe de plantão da enfermagem e médica.

\section{Coleta de dados}

A experiência positiva no TP e parto foi avaliada pela subescala do questionário de experiência e satisfação com o parto (QESP) desenvolvido e validado em português (Costa et al., 2004). A subescala utilizada foi referente a experiência positiva com o TP e parto, esta é constituída por 22 itens, relativos à confirmação de expectativas, autocontrole, autoconfiança, conhecimento, prazer e satisfação com a experiência de parto. O resultado da subescala obteve-se somando a pontuação dos itens, que varia de 22 a 88, quanto maior a pontuação melhor foi a experiência positiva com o TP e parto. A experiência positiva foi distribuída em muito ruim ( 22 pontos), baixa ( 23 a 44 pontos), moderada ( 45 a 66 pontos) e alta (> 66 pontos).

Foram coletadas variáveis sociodemográficas, econômicas, histórico obstétrico e variáveis do parto. As variáveis sociodemográficas foram: local de moradia, idade, estado civil, etnia, escolaridade e profissão. A variável econômica foi renda familiar total e o número de moradores, incluindo a participante. Foi considerado como histórico obstétrico os dados sobre uso de substâncias na gravidez, comorbidades, gravidez desejada e gravidez planejada. As variáveis do parto foram: tempo do trabalho de parto, tempo do período expulsivo, posição adotada no período expulsivo, laceração, grau de laceração, uso de ocitocina, rompimento artificial de membranas, manobras de Hamilton e de Kristeller, sangramento intenso, período expulsivo prolongado, uso de medicação.

Além disso, também foram incluídas nestes estudo as variáveis dos neonatos: peso, estatura, perímetro cefálico (PC), 
Perímetro torácico (PT), escore de Apgar (Apgar, 2015)

Com relação às substâncias na gravidez foram considerados álcool e cigarro. Quanto às comorbidades foram consideradas diabetes mellitus 1 e 2, diabetes mellitus gestacional, hipertensão arterial, eclâmpsia, pré-eclâmpsia, infecção do trato urinário (ITU), alergia, anemia e depressão.

\section{Análise estatística}

As variáveis foram descritas por meio de frequência relativa, absoluta, média e desvio padrão (dados paramétricos) e mediana e intervalo de confiança de 95\% (IC 95\%) (dados não paramétricos). Para verificar a normalidade dos dados numéricos foi utilizado o teste de Shapiro-Wilk. Os dados paramétricos foram analisados por meio do teste $t$ não-pareado e os não-paramétricos por meio do teste Mann-Whitney. Os dados categóricos foram analisados por meio do teste Exato de Fisher, para verificar a diferença entre os grupos, além disso foi estimado o Risco Relativo (RR) e IC 95\%. Foi considerado estatisticamente significativo os valores de $p \leq 0,05$.

\section{Resultados}

A idade das gestantes não diferiu entre os grupos (GI: 21 anos [IC 95\%: 9,31; 34,76]; GC: 22,5 anos [IC 95\%: 11,38; 36,23]; U: 17,00; $\mathrm{p}=0,075)$. A Tabela 1 demonstra os dados socioeconômicos e demográficos nos grupos, sendo que não houve diferença significativa.

A experiência positiva no TP e parto está apresentada na Tabela 2. Ambos os grupos apresentaram experiência positiva moderada, contudo, observou-se que as mulheres do GC se sentiram mais confiantes durante o trabalho de parto e as mulheres que realizaram fisioterapia consideraram-se um membro útil e cooperativo com a equipe médica que a acompanhou durante o parto.

A Tabela 3 apresenta os fatores maternos. Quando analisadas as características maternas da amostra, observou-se que as mulheres direcionadas ao GI apresentaram significativamente mais ITU que o GC.

Os dados obstétricos estão apresentados na Tabela 4. No GI 80,9\% eram primíparas, já no GC às primíparas foram 54,5\%. Observa-se que mulheres primíparas apresentaram associação com a presença de laceração (RR: 2,05 [IC 95\%: 1,08; 3,91]; p = 0,007) e houve associação entre ITU e laceração na população geral (RR: 2,02; IC: 1,20; 3,39; p: = 0,003). Ademais, mulheres que sofreram laceração tiveram um tempo de trabalho de parto maior (laceração(+): 18,50 horas [IC 95\%: 11,33; 39,79]; laceração(-): 6,00 [IC 95\%: -5,052; 33,95]; U: 78,00; p = 0,013). Com isso, o GI apresentou maior risco de laceração que o grupo GC e o tempo de trabalho de parto foi maior no GI (GI: 24 horas [IC 95\%: 10,48; 43,92]; GC: 7 horas [IC 95\%: $1,02 ; 32,73]$; U: 93,50; $p=0,015)$.

A Tabela 5 descreve os dados dos neonatos, não sendo observadas diferenças significativas entre os grupos. 
Tabela 1. Dados socioeconômicos e demográficos do grupo intervenção (GI) e controle (GC).

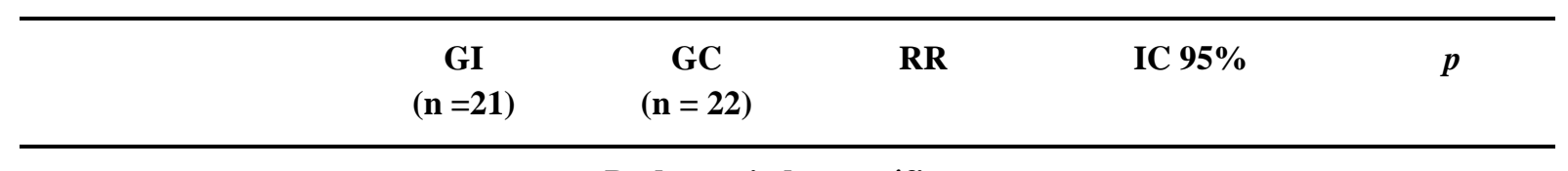

\section{Dados sociodemográficos}

\section{Local de moradia}

\begin{tabular}{|c|c|c|c|c|}
\hline Rural & $16(76,2)$ & $12(54,5)$ & 1,40 & 0,$89 ; 2,19$ \\
\hline Urbano & $4(19)$ & $7(31,8)$ & 0,60 & 0,$20 ; 1,75$ \\
\hline Não informado & $1(4,8)$ & $3(13,6)$ & 0,35 & 0,$04 ; 3,10$ \\
\hline
\end{tabular}

\section{Estado civil}

Casado

$3(14,3)$

$2(9,1)$

1,57

0,$29 ; 8,49$

0,477

Solteiro

$7(33,3)$

$7(31,8)$

1,05

0,$44 ; 2,48$

0,413

União estável

$11(52,4) \quad 13(59,1)$

0,89

0,$52 ; 1,51$

0,446

\section{Etnia}

$2(9,5)$

$0(0)$

2,15

0,$71 ; 12,00$

0,232

Parda

$18(85,7)$

$18(81,8)$

1,05

0,$81 ; 1,36$

0,473

Preta

$1(4,8)$

$4(18,2)$

0,26

0,$03 ; 2,16$

0,185

Escolaridade

Sem escolaridade

$0(0)$

$1(4,5)$

0,00

0,$00 ; 1,71$

0,511

Fundamental

4(19)

$6(27,3)$

0,70

0,$23 ; 2,13$

0,390

incompleto

Fundamental

$0(0)$

$1(4,5)$

0,00

0,$00 ; 1,71$

0,511

completo

Médio incompleto

$6(28,6)$

$4(18,2)$

1,57

0,$52 ; 4,79$

0,328

Médio completo

$9(42,9)$

$9(40,9)$

1,05

0,$52 ; 2,12$

0,428

Superior completo

$2(9,5)$

$1(4,5)$

1,40

0,$42 ; 2,46$

0,483

\section{Profissão}

ACS

Auxiliar Pedagógica

Contadora

Decoradora
$1(4,8)$

$0(0)$

$1(4,5)$

$1(4,8)$

$1(4,8)$

O(0)

$0(0)$

0(0)
2,10

0,00

2,00

2,00
0,$42 ; 23,07$

0,488

0,$00 ; 1,71$

$>0,999$

0,$41 ; 5,41$

$>0,999$

0,$41 ; 5,41$

$>0,999$ 


\begin{tabular}{|c|c|c|c|c|c|}
\hline Desempregada & $0(0)$ & $1(4,5)$ & 0,00 & 0,$00 ; 1,71$ & $>0,999$ \\
\hline Do lar & $9(42,9)$ & $11(50)$ & 0,86 & 0,$45 ; 1,64$ & 0,435 \\
\hline Estudante & $1(4,8)$ & $3(13,6)$ & 0,35 & 0,$04 ; 3,10$ & 0,316 \\
\hline Lavradora & $5(23,8)$ & $4(18,2)$ & 1,31 & 0,$41 ; 4,22$ & 0,468 \\
\hline Manicure & $2(9,5)$ & $0(0)$ & 2,158 & 0,$71 ; 12,00$ & 0,232 \\
\hline Marisqueira & $0(0)$ & $1(4,5)$ & 0,00 & 0,$00 ; 1,71$ & $>0,999$ \\
\hline Vendedora & $1(4,8)$ & $0(0)$ & 2,10 & 0,$42 ; 23,07$ & 0,488 \\
\hline Não informado & $0(0)$ & $1(4,5)$ & - & - & - \\
\hline \multicolumn{6}{|c|}{ Dados econômicos } \\
\hline \multicolumn{6}{|l|}{$\begin{array}{c}\text { Renda familiar } \\
\text { Total }\end{array}$} \\
\hline Nenhuma & $2(9,5)$ & $1(4,5)$ & 1,40 & 0,$42 ; 2,45$ & 0,483 \\
\hline Não sabe & $2(9,5)$ & $4(18,2)$ & 0,52 & 0,$11 ; 2,57$ & 0,352 \\
\hline $\begin{array}{l}\text { Menos de } 1 \text { salário } \\
\text { mínimo }\end{array}$ & $11(52,4)$ & $12(54,5)$ & 0,96 & 0,$55 ; 1,68$ & 0,435 \\
\hline $\begin{array}{l}\text { De } 1 \text { a } 3 \text { salários } \\
\text { mínimo }\end{array}$ & $6(28,6)$ & $5(22,7)$ & 1,26 & 0,$45 ; 3,5$ & 0,464 \\
\hline \multicolumn{6}{|l|}{ Total de moradores } \\
\hline De 1 a 3 Pessoas & $9(42,9)$ & $8(36,4)$ & 1,18 & 0,$56 ; 2,47$ & 0,450 \\
\hline De 4 a 7 Pessoas & $11(52,4)$ & $14(63,6)$ & 0,82 & 0,$49 ; 1,38$ & 0,330 \\
\hline De 8 a 10 Pessoas & $1(4,8)$ & $0(0)$ & 2,10 & 0,$42 ; 23,07$ & 0,488 \\
\hline
\end{tabular}

RR: Risco relativo; IC 95\%: Intervalo de Confiança; ACS: Agente Comunitário de Saúde. ${ }^{*} \mathrm{p}<0,05$, teste exato de Fisher. Fonte: Autores. 
Tabela 2. Experiência positiva no trabalho de parto do grupo intervenção (GI) e controle (GC).

\begin{tabular}{|c|c|c|c|c|}
\hline Itens & $\begin{array}{c}\text { GI } \\
(\mathbf{n}=\mathbf{2 1})\end{array}$ & $\begin{array}{c}\text { GC } \\
(\mathbf{n}=\mathbf{2 2})\end{array}$ & $\mathbf{U} / \mathbf{t}$ & $\mathbf{p}$ \\
\hline $\begin{array}{l}\mathrm{O} \mathrm{TP} \text { decorreu de encontro com suas } \\
\text { expectativas? }\end{array}$ & $\begin{array}{c}2,00(1,57 \\
2,43)\end{array}$ & $\begin{array}{l}2,50(2,05 \\
2,95)\end{array}$ & 168,0 & 0,057 \\
\hline $\begin{array}{l}\mathrm{O} \text { P ocorreu de encontro com suas } \\
\text { expectativas? }\end{array}$ & $\begin{array}{c}3,00(2,20 \\
3,04)\end{array}$ & $\begin{array}{c}3,00(2,26 \\
3,10)\end{array}$ & 217,5 & 0,365 \\
\hline $\begin{array}{l}\text { A dor que sentiu no TP foi de acordo com suas } \\
\text { expectativas? }\end{array}$ & $\begin{array}{l}2,00(1,68 \\
2,70)\end{array}$ & $\begin{array}{c}2,00(1,70 \\
2,39)\end{array}$ & 224,0 & 0,434 \\
\hline $\begin{array}{l}\text { A dor que sentiu no } \mathrm{P} \text { foi de acordo com suas } \\
\text { expectativas? }\end{array}$ & $\begin{array}{c}2,00(1,67 \\
2,52)\end{array}$ & $\begin{array}{c}2,00(1,90 \\
2,83)\end{array}$ & 198,0 & 0,204 \\
\hline $\begin{array}{l}\text { Sentiu que tinha a situação sob controle } \\
\text { durante o TP? }\end{array}$ & $\begin{array}{c}2,00(1,71 \\
2,29)\end{array}$ & $\begin{array}{c}2,00(1,98 ; \\
2,83)\end{array}$ & 183,0 & 0,077 \\
\hline $\begin{array}{l}\text { Sentiu que tinha a situação sob controle } \\
\text { durante o P? }\end{array}$ & $\begin{array}{c}2,00(1,81 \\
2,47)\end{array}$ & $\begin{array}{c}2,00(1,93 \\
2,64)\end{array}$ & 195,0 & 0,243 \\
\hline Sentiu-se confiante durante o TP? & $\begin{array}{c}2,00(1,86 \\
2,62)\end{array}$ & $\begin{array}{c}3,00(2,42 \\
3,04)\end{array}$ & 159,0 & $0,031 *$ \\
\hline Sentiu-se confiante durante o P? & $\begin{array}{l}3,00(2,13 \\
3,02)\end{array}$ & $\begin{array}{c}3,00(2,36 \\
3,17)\end{array}$ & 198,0 & 0,281 \\
\hline $\begin{array}{l}\text { Tinha conhecimento de todos } \\
\text { acontecimentos relativos ao TP? }\end{array}$ & $\begin{array}{c}2,00(1,64 \\
2,55)\end{array}$ & $\begin{array}{c}2,00(1,68 \\
2,60)\end{array}$ & 227,5 & 0,470 \\
\hline $\begin{array}{l}\text { Tinha conhecimento de todos } \\
\text { acontecimentos relativos ao P? }\end{array}$ & $\begin{array}{l}2,00(1,36 \\
2,04)\end{array}$ & $\begin{array}{l}2,00(1,68 \\
2,50)\end{array}$ & 169,0 & 0,087 \\
\hline $\begin{array}{l}\text { Tinha conhecimento de todos os } \\
\text { acontecimentos relativos ao PP? }\end{array}$ & $\begin{array}{c}2,00(1,41 \\
2,21)\end{array}$ & $\begin{array}{c}2,00(1,61 \\
2,39)\end{array}$ & 204,0 & 0,246 \\
\hline $\begin{array}{l}\text { Em algum momento sentiu prazer ou satisfação } \\
\text { durante o TP? }\end{array}$ & $\begin{array}{c}1,00(1,31 \\
2,21)\end{array}$ & $\begin{array}{c}2,00(1,60 \\
2,49)\end{array}$ & 190,0 & 0,147 \\
\hline $\begin{array}{l}\text { Em algum momento sentiu prazer ou satisfação } \\
\text { durante o P? }\end{array}$ & $\begin{array}{c}2,00(1,47 \\
2,33)\end{array}$ & $\begin{array}{l}2,00(1,42 \\
2,04)\end{array}$ & 215,0 & 0,341 \\
\hline $\begin{array}{l}\text { Considera que foi um membro útil e } \\
\text { cooperativo com a equipe médica que a } \\
\text { acompanhou durante o TP? }\end{array}$ & $\begin{array}{c}3,00(3,08 \\
3,68)\end{array}$ & $\begin{array}{c}3,00(2,73 \\
3,45)\end{array}$ & 186,0 & 0,122 \\
\hline $\begin{array}{l}\text { Considera que foi um membro útil e } \\
\text { cooperativo com a equipe médica que a } \\
\text { acompanhou durante o P? }\end{array}$ & $\begin{array}{c}4,00(3,04 \\
3,72)\end{array}$ & $\begin{array}{c}3,00(2,39 \\
3,16)\end{array}$ & 141,5 & $0,011 *$ \\
\hline $\begin{array}{l}\text { Considera que foi um membro útil e } \\
\text { cooperativo com a equipe médica que a } \\
\text { acompanhou logo após o parto? }\end{array}$ & $\begin{array}{c}3,00(2,73 \\
3,47)\end{array}$ & $\begin{array}{c}3,00(2,55 \\
3,18)\end{array}$ & 185,5 & 0,174 \\
\hline
\end{tabular}




\begin{tabular}{|c|c|c|c|c|}
\hline $\begin{array}{l}\text { Está satisfeita com a forma como decorreu o } \\
\text { TP? }\end{array}$ & $\begin{array}{l}3,00(2,53 \\
3,28)\end{array}$ & $\begin{array}{l}2,00(2,26 ; \\
3,10)\end{array}$ & 194,0 & 0,175 \\
\hline Está satisfeita com a forma como decorreu o P? & $\begin{array}{l}3,00(2,50 \\
3,31)\end{array}$ & $\begin{array}{l}3,00(2,49 \\
3,23)\end{array}$ & 221,0 & 0,404 \\
\hline $\begin{array}{l}\text { Está satisfeita com a forma como decorreu o } \\
\text { PP? }\end{array}$ & $\begin{array}{l}3,00(2,53 \\
3,19)\end{array}$ & $\begin{array}{l}2,50(2,34 \\
3,20)\end{array}$ & 216,0 & 0,354 \\
\hline Está satisfeita com o tempo que demorou o TP? & $\begin{array}{c}2,00(1,74 \\
2,73)\end{array}$ & $\begin{array}{l}2,00(1,94 \\
2,76)\end{array}$ & 190,5 & 0,297 \\
\hline Está satisfeita com o tempo que demorou o P? & $\begin{array}{l}3,00(2,25 \\
3,08)\end{array}$ & $\begin{array}{c}2,00(1,99 \\
2,92)\end{array}$ & 200,5 & 0,223 \\
\hline Está satisfeita com o tempo que demorou o PP? & $\begin{array}{l}2,00(2,20 \\
3,04)\end{array}$ & $\begin{array}{l}2,00(1,99 \\
2,55)\end{array}$ & 186,5 & 0,111 \\
\hline Satisfação positiva final & $52,95 \pm 10,61$ & $53,45 \pm 9,23$ & 0,165 & 0,435 \\
\hline
\end{tabular}

TP: Trabalho de Parto; P: Parto; PP: Pós-Parto. *p<0,05, teste t ou de Mann-Whitney. Fonte: Autores.

Tabela 3. Fatores maternos dos grupos intervenção (GI) e controle (GC).

\begin{tabular}{|c|c|c|c|c|c|}
\hline & $\begin{array}{c}\text { GI } \\
(\mathbf{n}=\mathbf{2 1})\end{array}$ & $\begin{array}{c}\text { GC } \\
(n=22)\end{array}$ & $\mathbf{R} \mathbf{R}$ & IC $95 \%$ & $p$ \\
\hline \multicolumn{6}{|c|}{ Uso de substâncias } \\
\hline Álcool & $1(4,8)$ & $2(8,7)$ & 0,66 & 0,$12 ; 1,78$ & 0,467 \\
\hline Cigarro & $0(0)$ & $1(4,3)$ & 0,00 & 0,$00 ; 1,79$ & $>0,999$ \\
\hline Nenhum & $20(95,2)$ & $20(87)$ & 1,50 & 0,$56 ; 8,27$ & 0,333 \\
\hline \multicolumn{6}{|c|}{ Comorbidades } \\
\hline DM Gestacional & $1(4,7)$ & $0(0)$ & 2,09 & 0,$42 ; 5,65$ & 0,489 \\
\hline ITU & $14(66,6)$ & $8(36,3)$ & 1,91 & 1,$00 ; 3,65$ & $0,030 *$ \\
\hline Alergia & $0(0)$ & $1(4,5)$ & 0,00 & 0,$00 ; 1,78$ & $>0,999$ \\
\hline Anemia & $0(0)$ & $1(4,5)$ & 0,00 & 0,$00 ; 1,78$ & $>0,999$ \\
\hline Depressão & $0(0)$ & $1(4,5)$ & 0,00 & 0,$00 ; 1,78$ & $>0,999$ \\
\hline Não possui & $7(33,3)$ & $13(59)$ & 0,59 & 0,$29 ; 1,20$ & 0,109 \\
\hline \multicolumn{6}{|c|}{ Gravidez } \\
\hline Desejada & $17(80,9)$ & $16(16,7)$ & 1,11 & 0,$80 ; 1,55$ & 0,390 \\
\hline Planejada & $10(47,6)$ & $7(31,8)$ & 1,50 & 0,$70 ; 3,20$ & 0,227 \\
\hline
\end{tabular}

RR: Risco relativo; IC 95\%: Intervalo de Confiança; ITU: Infecção do Trato Urinário; DM: Diabete Mellitus. *p<0,05, teste exato de Fisher. Fonte: Autores. 
Tabela 4. Dados obstétricos dos grupos intervenção (GI) e controle (GC).

\begin{tabular}{|c|c|c|c|c|c|}
\hline & $\begin{array}{c}\text { GI } \\
(\mathbf{n}=21)\end{array}$ & $\begin{array}{c}\text { GC } \\
(\mathbf{n}=\mathbf{2 2})\end{array}$ & $\mathbf{R R}$ & IC 95\% & $p$ \\
\hline \multicolumn{6}{|l|}{$\begin{array}{c}\text { Posição adotada no } \\
\text { parto }\end{array}$} \\
\hline Cesária & $1(4,8)$ & $0(0)$ & 2,15 & 0,$43 ; 3,00$ & 0,476 \\
\hline 4 apoios & $1(4,8)$ & $0(0)$ & 2,15 & 0,$43 ; 3,00$ & 0,476 \\
\hline Decúbito lateral & $1(4,8)$ & $0(0)$ & 2,15 & 0,$43 ; 3,00$ & 0,476 \\
\hline Decúbito dorsal & $6(28,6)$ & $10(45,4)$ & 0,63 & 0,$28 ; 1,42$ & 0,203 \\
\hline Litotomia & $6(28,6)$ & $11(50)$ & 0,57 & 0,$26 ; 1,27$ & 0,130 \\
\hline Semi sentada & $3(14,3)$ & $1(4,54)$ & 3,14 & 0,$35 ; 27,88$ & 0,283 \\
\hline Semi deitada & $1(4,8)$ & $0(0)$ & 2,15 & 0,$43 ; 3,00$ & 0,476 \\
\hline Sentada & $1(4,8)$ & $0(0)$ & 2,15 & 0,$43 ; 3,00$ & 0,476 \\
\hline Não informado & $1(4,8)$ & $0(0)$ & - & - & - \\
\hline Laceração & $20(95,2)$ & $8(36,3)$ & 2,62 & 1,$49 ; 4,59$ & $<0,0001 *$ \\
\hline \multicolumn{6}{|l|}{ Grau de laceração } \\
\hline 1 & $3(14,2)$ & $0(0)$ & 1,40 & 0,$60 ; 1,77$ & 0,549 \\
\hline 2 & $9(42,8)$ & $0(0)$ & 1,40 & 0,$95 ; 3,24$ & 0,158 \\
\hline Não Informado & $8(38)$ & $8(36,3)$ & - & - & - \\
\hline \multicolumn{6}{|l|}{$\begin{array}{c}\text { Intervenções durante } \\
\text { o parto }\end{array}$} \\
\hline Uso de Ocitocina & $13(61,9)$ & $12(54,5)$ & 0,97 & 0,$50 ; 1,88$ & 0,444 \\
\hline Amniotomia & $16(76,1)$ & $14(63,6)$ & 1,02 & 0,$57 ; 1,83$ & 0,438 \\
\hline Manobra de Hamilton & $14(66,6)$ & $14(63,3)$ & 0,89 & 0,$48 ; 1,65$ & 0,447 \\
\hline Manobra de Kristeller & $1(4,7)$ & $1(4,5)$ & 1,02 & 0,$19 ; 2,18$ & 0,262 \\
\hline Uso de outra medicação & $0(0)$ & $1(4,5)$ & 0,00 & 0,$00 ; 1,55$ & 0,477 \\
\hline \multicolumn{6}{|l|}{$\begin{array}{l}\text { Complicações no } \\
\text { parto }\end{array}$} \\
\hline Sangramento Intenso & $1(4,7)$ & $0(0)$ & 1,89 & 0,$38 ; 4,60$ & $>0,999$ \\
\hline $\begin{array}{ll}\text { Período } & \text { expulsivo } \\
\text { prolongado } & \end{array}$ & $1(4,7)$ & $0(0)$ & 1,89 & 0,$38 ; 4,60$ & $>0,999$ \\
\hline
\end{tabular}

RR: Risco relativo; IC95\%: Intervalo de Confiança. ${ }^{*} \mathrm{p}<0,05$, teste exato de Fisher. Fonte: Autores. 
Tabela 5. Medidas neonatais dos grupos de intervenção (GI) e controle (GC).

\begin{tabular}{|c|c|c|c|c|}
\hline & $\begin{array}{c}\text { GI } \\
(\mathbf{n}=\mathbf{2 1})\end{array}$ & $\begin{array}{c}\text { GC } \\
(\mathbf{n}=\mathbf{2 2})\end{array}$ & $p$ & Teste \\
\hline Peso (g) & $3285(66,12 ; 246,12)$ & $3295(66,12 ; 246,12)$ & 0,449 & $\mathrm{U}=174,500$ \\
\hline Estatura $(\mathrm{cm})$ & $49(36,01 ; 61,98)$ & $49(35,44 ; 62,13)$ & 0,331 & $\mathrm{U}=17,500$ \\
\hline Perímetro Torácico $(\mathrm{cm})$ & $33(19,96 ; 45,93)$ & $32(19,49 ; 46,19)$ & 0,277 & $\mathrm{U}=169,000$ \\
\hline Perímetro Cefálico (cm) & $33,37 \pm 1,22$ & $33,52 \pm 1,40$ & 0,362 & $\mathrm{t}=-0,355$ \\
\hline Escala de Apgar & $9(-4,33 ; 21,63)$ & $9(-4,76 ; 21,92)$ & 0,331 & $\mathrm{U}=174,500$ \\
\hline
\end{tabular}

${ }^{*} \mathrm{p}<0,05$, Teste $t$ (paramétrica) e Mann-Whitney (não-paramétrica). Fonte: Autores.

\section{Discussão}

O estudo propôs avaliar o efeito da fisioterapia sobre as experiências positivas no trabalho de parto e parto em um hospital de referência materno-infantil na região Amazônica oriental. Observou-se que ambos os grupos apresentaram experiências positivas moderadas, porém, não houve diferença estatística entre os grupos. Este desfecho confronta a literatura, pois a prática de exercício e a realização de massagem durante o trabalho de parto demonstra influência positiva sobre as experiências deste evento. (Çevik \& Karaduman, 2019; Slade et al., 1993; Unalmis et al., 2017)

Entretanto, a fisioterapia apresentou efeito positivo ao mostrar que as mulheres do grupo intervenção se consideraram mais participativas com a equipe médica durante o parto, aspecto que pode contribuir para melhores experiências com o nascimento. Pois, a boa qualidade de atendimento por parte dos profissionais da saúde, especificamente por percepções de competência técnica, empatia e suporte, além do envolvimento da mulher nas tomadas de decisões e o maior número de interações médico-paciente são fatores relevantes para experiência positiva com o parto. Sendo o cuidado da equipe, respeito pelo parturiente e infraestrutura as dimensões mais importantes para a mulher prever o seu nível de satisfação (Bell et al., 2018; Jha et al., 2017; Pantoja et al., 2019).

Vale salientar que quando o cuidado profissional é fornecido em altos níveis de intervenções, as mulheres podem ficar menos satisfeitas (Ferrer et al., 2016; Weeks et al., 2017). Fato este que pode ter contribuído para a avaliação de experiência positiva moderada, pois constatou-se um alto número de intervenções médicas, como o uso de ocitocina, rompimento artificial de membranas e manobras de Hamilton e Kristeller.

Outros fatores que podem contribuir para insatisfação com a parto são a raça, a paridade e a duração do trabalho de parto. Em estudo de Hamm et al. (2019), mulheres autodeclaradas negras, primíparas e que tiveram um maior tempo de trabalho de parto tiveram maior probabilidade de serem insatisfeitas com o parto. A maior parte da amostra deste estudo foi composta por mulheres negras e pardas. Quanto à paridade, a distribuição de primíparas e multíparas foi heterogênea entre os grupos, sendo que a maior parte das mulheres que receberam fisioterapia eram primíparas, evento este que pode ter contribuído para maior tempo de trabalho de parto.

A literatura mostra que mulheres com idade maior que 22 anos apresentam maiores níveis de satisfação com parto (Jha et al., 2017), bem como as que têm gestação planejada (Salmon \& Drew, 1992). Neste estudo as parturientes do grupo intervenção apresentaram mediana de 21 anos e o grupo controle de 23 anos e menos da metade das pacientes planejaram a gravidez. 
Outro desfecho encontrado neste estudo foi a laceração perineal, constatou-se relação da laceração com a primiparidade. Corroborando com Peppe et al. (2018), que associa este evento com a paridade e a idade da mãe, em seu estudo mulheres primigestas e jovens tiveram maior propensão a sofrerem trauma perineal, não havendo associação entre os dados antropométricos do RN e a posição de parto, vertical ou horizontal (Peppe et al., 2018). Outro estudo analisou os fatores que aumentam o número de lesão perineal e constatou que o uso de ocitocina, manobra de valsalva e a posição de parto horizontal provocam maior taxa de laceração (Tunestveit et al., 2018). Além disso, nesta pesquisa houve associação com tempo de TP e ocorrência de ITU na gestação, resultado nunca descrito em estudos anteriores.

O estudo apresentou limitações para afirmar o real efeito da fisioterapia sobre a laceração perineal e tempo de trabalho de parto, devido a não-randomização da amostra, sendo o grupo intervenção composto em sua maioria por primigestas, evento este que apresentou correlação com presença de laceração perineal. Ademais, a laceração associou-se ao maior tempo de trabalho de parto e presença de ITU, comorbidade que foi mais prevalente em mulheres que realizaram fisioterapia. Outra limitação foi o período de realização da coleta, pois ocorreu em meio a pandemia pelo covid-19, momento este que pode influenciar nos aspectos emocionais das parturientes, pelo receio em torno do risco de contágio dela e do RN.

\section{Conclusão}

As mulheres deste estudo apresentaram experiência positiva moderada com o trabalho de parto e parto e as mulheres que realizaram fisioterapia tiveram como experiência positiva o fato de se considerarem um membro útil e cooperativo com a equipe médica que a acompanhou durante o parto. Além disso, observou-se que a primiparidade, principal evento da amostra no grupo intervenção, foi associada a laceração, sendo esta aumentada junto com o tempo de trabalho de parto e presença de ITU.

Os resultados obtidos podem embasar novos estudos sobre fatores de risco e consequências da laceração, assim como desenvolver estratégias para sua prevenção. Por fim, sugerimos que sejam realizados novos estudos visando verificar os efeitos entre a fisioterapia realizada ao longo do período pré-natal e apenas no trabalho de parto.

\section{Referências}

Abbasi, S., Chuang, C. H., Dagher, R., Zhu, J., \& Kjerulff, K. (2013). Unintended pregnancy and postpartum depression among first-time mothers. Journal of women's health (2002), 22(5), 412-416. https://doi.org/10.1089/jwh.2012.3926

Akköz Çevik, S., \& Karaduman, S. (2020). The effect of sacral massage on labor pain and anxiety: A randomized controlled trial. Japan journal of nursing science: JJNS, 17(1), e12272. https://doi.org/10.1111/jjns. 12272

Apgar V. (2015). A Proposal for a New Method of Evaluation of the Newborn Infant. Originally published in July 1953, volume 32, pages 250-259. Anesthesia and analgesia, 120(5), 1056-1059. https://doi.org/10.1213/ANE.0b013e31829bdc5c

Baracho E. (2018). Fisioterapia aplicada à saúde da mulher. Guanabara Koogan.

Bell, A. F., Andersson, E., Goding, K., \& Vonderheid, S. C. (2018). The birth experience and maternal caregiving attitudes and behavior: A systematic review. Sexual \& reproductive healthcare: official journal of the Swedish Association of Midwives, 16, 67-77. https://doi.org/10.1016/j.srhc.2018.02.007

Conesa Ferrer, M. B., Canteras Jordana, M., Ballesteros Meseguer, C., Carrillo García, C., \& Martínez Roche, M. E. (2016). Comparative study analysing women's childbirth satisfaction and obstetric outcomes across two different models of maternity care. BMJ open, 6(8), e011362. https://doi.org/10.1136/bmjopen-2016-011362

Costa R., Figueiredo, B., Pacheco, A. P., Marques, A., \& Pais, A. Questionário de Experiência e Satisfação com o Parto (QESP). (2004) Psicologia, Saúde \& Doenças, 5(2), 159-187. http://hdl.handle.net/1822/5850

Downe, S., Finlayson, K., Oladapo, O. T., Bonet, M., \& Gülmezoglu, A. M. (2018). What matters to women during childbirth: A systematic qualitative review. PloS one, 13(4), e0194906. https://doi.org/10.1371/journal.pone.0194906

Estrela, C. (2018). Metodologia científica: ciência, ensino, pesquisa. Artes Médicas. 
Faul, F., Erdfelder, E., Lang, A. G., \& Buchner, A. (2007). G*Power 3: a flexible statistical power analysis program for the social, behavioral, and biomedical sciences. Behavior research methods, 39(2), 175-191. https://doi.org/10.3758/bf03193146

Hamm, R. F., Srinivas, S. K., \& Levine, L. D. (2019). Risk factors and racial disparities related to low maternal birth satisfaction with labor induction: a prospective, cohort study. BMC pregnancy and childbirth, 19(1), 530. https://doi.org/10.1186/s12884-019-2658-z

Jha, P., Larsson, M., Christensson, K., \& Skoog Svanberg, A. (2017). Satisfaction with childbirth services provided in public health facilities: results from a cross- sectional survey among postnatal women in Chhattisgarh, India. Global health action, 10(1), 1386932. https://doi.org/10.1080/16549716.2017.1386932

Nakano, A. R., Bonan, C., \& Teixeira, L. A. (2016). Cesárea, aperfeiçoando a técnica e normatizando a prática: uma análise do livro Obstetrícia, de Jorge de Rezende. História, Ciências, Saúde-Manguinhos, 23(1), 155-172. https://doi.org/10.1590/S0104-59702016000100010

Nilvér, H., Begley, C., \& Berg, M. (2017). Measuring women's childbirth experiences: a systematic review for identification and analysis of validated instruments. BMC pregnancy and childbirth, 17(1), 203. https://doi.org/10.1186/s12884-017-1356-y

Ministério da Saúde. (2017). Diretrizes nacionais de assistência ao parto normal: versão resumida. Secretaria de Ciência, Tecnologia e Insumos Estratégicos, Departamento de Gestão e Incorporação de Tecnologias https://bvsms.saude.gov.br/bvs/publicacoes/diretrizes_nacionais_assistencia_parto_normal.pdf

Pantoja, L., Weeks, F. H., Ortiz, J., Cavada, G., Foster, J., \& Binfa, L. (2020). Dimensions of childbirth care associated with maternal satisfaction among lowrisk Chilean women. Health care for women international, 41(1), 89-100. https://doi.org/10.1080/07399332.2019.1590360

Peppe, M. V., Stefanello, J., Infante, B. F., Kobayashi, M. T., Baraldi, C. O., \& Brito, L. (2018). Perineal Trauma in a Low-risk Maternity with High Prevalence of Upright Position during the Second Stage of Labor. Trauma perineal em uma maternidade de baixo risco com alta prevalência de parto vertical durante o período expulsivo. Revista brasileira de ginecologia e obstetrícia : revista da Federação Brasileira das Sociedades de Ginecologia e Obstetrícia, 40(7), 379-383. https://doi.org/10.1055/s-0038-1666810

Salmon, P., \& Drew, N. C. (1992). Multidimensional assessment of women's experience of childbirth: relationship to obstetric procedure, antenatal preparation and obstetric history. Journal of psychosomatic research, 36(4), 317-327. https://doi.org/10.1016/0022-3999(92)90068-D

Slade, P., MacPherson, S. A., Hume, A., \& Maresh, M. (1993). Expectations, experiences and satisfaction with labour. The British journal of clinical psychology, 32(4), 469-483. https://doi.org/10.1111/j.2044-8260.1993.tb01083.x

Tunestveit, J. W., Baghestan, E., Natvig, G. K., Eide, G. E., \& Nilsen, A. B. V. (2018). Factors associated with obstetric anal sphincter injuries in midwife-led birth: A cross sectional study. Midwifery, 62, 264-272. https://doi.org/10.1016/j.midw.2018.04.012

Unalmis Erdogan, S., Yanikkerem, E., \& Goker, A. (2017). Effects of low back massage on perceived birth pain and satisfaction. Complementary therapies in clinical practice, 28, 169-175. https://doi.org/10.1016/j.ctcp.2017.05.016

Weeks, F., Pantoja, L., Ortiz, J., Foster, J., Cavada, G., \& Binfa, L. (2017). Labor and Birth Care Satisfaction Associated With Medical Interventions and Accompaniment During Labor Among Chilean Women. Journal of midwifery \& women's health, 62(2), 196-203. https://doi.org/10.1111/jmwh.12499 